\title{
PROPAGATION IN A CIRCULAR WAVEGUIDE PERIODICALLY LOADED WITH DIELECTRIC DISKS
}

\author{
S. Amari, R. Vahldieck, J. Bornemann* and P.Leuchtmann \\ Swiss Federal Institute of Technology, ETH Zentrum, Gloriastrasse 35, CH - 8092 Zürich, \\ http://www.ifh.ee.ethz.ch. \\ *Department of Electrical and Computer Engineering \\ University of Victoria, BOX 3055 \\ Victoria, B.C. V8W 3P6 \\ http://www.ece.uvic.ca
}

\section{Introduction}

$\mathrm{P}$ ERIODIC structures have been investigated by many researchers due to their importance in slow wave and backward-wave devices [1], [2].

One of the salient features of propagation in periodic structures is the presence of passbands, where undamped waves propagate, separated by stopbands where no energy transport takes place. The spatial periodicity of the structure is reflected in the field distribution through the Floquet condition which we express in the following form

$$
F(z+p)=e^{-\theta p} F(z) .
$$

Here, $p$ is the period of the structure, $\theta$ is the propagation constant and $F(z)$ is a generic component of the electromagnetic field.

The analysis of propagation in periodically loaded waveguides requires the enforcement of the Floquet condition in addition to the other boundary conditions of the waveguide. Available methods of analysis of these structures are mainly based on expansions in space-harmonics to account for the Floquet condition [2]. Within this framework the propagation constants of the Floquet modes are determined as the zero of a determinant following iterative root-finding algorithms. Such an approach can be time consuming, especially when a large number of modes are needed, and suffers from the risk of missing physical solutions when two or more roots coincide. Simplifications of the approach, such as the surface impedance approach, are often used in investigating propagation in corrugated waveguides and horns, for example. An alternative approach consists in deriving equivalent lumped elements for the loading sections followed by a circuit analysis. Within this framework, the propagation constants are determined from the eigenvalues of a square matrix whose size is given by the number of accessible modes. The main shortcoming of this approach resides in its limitation to the branches of the fundamental mode for which the equivalent lumped element is derived in addition to the arbitrariness in determining the appropriate number of accessible modes.

In this paper, we present an analysis of propagation in a circular waveguide periodically loaded with dielectric disks. The approach is based on an extension of the Coupled-Integral-Equation Technique (CIET) [3]. The propagation constants are determined from the classical eigenvalues of a non-Hermitian matrix instead of a determinant thereby considerably reducing CPU times. The Floquet condition which is viewed as a priori information on the exact solution, is included in the integral equations from the outset. Other relevant a priori information, such as the edge conditions or other symmetries can also be accommodated through appropriate basis functions.

\section{Theory}

The structure under consideration is depicted in Figure 1. It consists of a lossless circular waveguide of radius $a$ periodically loaded with dielectric disks of radius $b$ and dielectric constant $\epsilon_{r}$. The thickness of the disks is $t$ and the period of the 
structure is $p$.

We focus attention only on the TE modes with no angular dependence. This situation is of practical interest in $\mathrm{TE}_{01 \delta}$ resonator filters. Due to the periodicity of the structure it is sufficient to concentrate one one unit cell.

In regions I (dielectric loaded) and II we expand the transverse components of the electromagnetic field in series of the normal modes, i.e,

$$
\begin{gathered}
E_{\phi}^{I}(\rho, z)=\sum_{m=1}^{\infty} \psi_{m}^{I}(\rho)\left[F_{m}^{I} e^{-j k_{z m}^{I} z}+B_{m}^{I} e^{j k_{z m}^{I} z}\right] \\
H_{\rho}^{I}(\rho, z)=\sum_{m=1}^{\infty} \psi_{m}^{I}(\rho) Y_{m}^{I}\left[F_{m}^{I} e^{-j k_{z m}^{I} z}-B_{m}^{I} e^{j k_{z m}^{I} z}\right] \\
E_{\phi}^{I I}(\rho, z)=\sum_{m=1}^{\infty} \psi_{m}^{I I}(\rho)\left[F_{m}^{I I} e^{-j k_{z m}^{I I} z}+B_{m}^{I I} e^{j k_{z m}^{I I} z}\right] \\
H_{\rho}^{I I}(\rho, z)=\sum_{m=1}^{\infty} \psi_{m}^{I I}(\rho) Y_{m}^{I I}\left[F_{m}^{I I} e^{-j k_{z m}^{I I} z}-B_{m}^{I I} e^{j k_{z m}^{I I} z}\right]
\end{gathered}
$$

Here, $k_{z m}^{i}$ is the propagation constant of mode $m$, $Y_{m}^{i}=\frac{k_{z m}^{i}}{\omega \mu_{0}}$ and $\psi_{m}^{i}(\rho)$ are the normal modes of the $\mathrm{i}^{\text {th }}$ region. The modal constants $F_{m}^{i}$ and $B_{m}^{i}$ are unknown expansion coefficients.

The boundary conditions of the problem can be written in the form

$$
\begin{gathered}
E_{\phi}^{I}(\rho, z=t)=E_{\phi}^{I I}(\rho, z=t), \quad 0 \leq \rho \leq a \quad(6) \\
H_{\rho}^{I}(\rho, z=t)=H_{\rho}^{I I}(\rho, z=t), \quad 0 \leq \rho \leq a \quad(7) \\
E_{\phi}^{I I}(\rho, z=p)=e^{-\theta p} E_{\phi}^{I}(\rho, z=0), \quad 0 \leq \rho \leq a \\
H_{\rho}^{I I}(\rho, z=p)=e^{-\theta p} H_{\rho}^{I}(\rho, z=0), \quad 0 \leq \rho \leq a
\end{gathered}
$$

Let us assume that the transverse electric field at $z=0, z=t$ and $z=p$ are given by three unknown functions $X_{1}(\rho), X_{2}(\rho)$ and $X_{3}(\rho)$, respectively. The Floquet condition on the electric field is satisfied by requiring the following relationship

$$
X_{3}(\rho)=e^{-\theta p} X_{1}(\rho) .
$$

To derive integral equations for the functions $X_{1}(\rho)$ and $X_{2}(\rho)$, we use the continuity of $E_{\phi}$ at $z=0$ and $z=t$ to express the modal expansion coefficients in terms of integrals of $X_{1}(\rho)$ and
$X_{2}(\rho)$ and then enforce the continuity of the magnetic field at $z=t$ and its Floquet condition. After some mathematical manipulations, we obtain the following two coupled integral equations

$$
\begin{aligned}
& \sum_{m=1}^{\infty} Y_{m}^{I I} \frac{\tilde{X}_{2}^{I I}(m) e^{\theta p}-\tilde{X}_{1}^{I I}(m) \cos \left(k_{z m}^{I I} s\right)}{\sin \left(k_{z m}^{I I} s\right)} \psi_{m}^{I I} \\
= & \sum_{m=1}^{\infty} Y_{m}^{I} \frac{\tilde{X}_{1}^{I}(m) \cos \left(k_{z m}^{I} t\right)-\tilde{X}_{2}^{I}(m)}{\sin \left(k_{z m}^{I} t\right)} \psi_{m}^{I}(\rho),
\end{aligned}
$$

and

$$
\begin{aligned}
& \sum_{m=1}^{\infty} Y_{m}^{I I} \frac{\tilde{X}_{2}^{I I}(m) \cos \left(k_{z m}^{I I} s\right)-e^{-\theta p} \tilde{X}_{1}^{I I}(m)}{\sin \left(k_{z m}^{I I} s\right)} \psi_{m}^{I I} \\
& =\sum_{m=1}^{\infty} Y_{m}^{I} \frac{\tilde{X}_{1}^{I}(m)-\tilde{X}_{2}^{I}(m) \cos \left(k_{z m}^{I} t\right)}{\sin \left(k_{z m}^{I} t\right)} \psi_{m}^{I}(\rho)
\end{aligned}
$$

Here, $s=p-t$ and

$$
\tilde{X}^{i}(m)=\int_{0}^{a} \rho d \rho \psi_{m}^{i}(\rho) X^{i}(\rho), \quad i=I, I I .
$$

These coupled integral equations are solved by the moment method starting from expansions of $X_{1}(\rho)$ and $X_{2}(\rho)$ in series of basis functions

$$
\begin{aligned}
& X_{1}(\rho)=\sum_{i=1}^{M} c_{i} Q_{i}(\rho) \\
& X_{2}(\rho)=\sum_{i=1}^{M} d_{i} Q_{i}(\rho)
\end{aligned}
$$

Using these expansions in the two coupled integral equations, and applying Galerkin's method, we get the following matrix eigenvalue equation

$$
\left(\left[\begin{array}{cc}
A & C \\
C & A
\end{array}\right]+\left[\begin{array}{cc}
D e^{-\theta p} & 0 \\
0 & D e^{\theta p}
\end{array}\right]\right)\left[\begin{array}{l}
c \\
d
\end{array}\right]=0
$$

The entries of the matrices are given by

$$
\begin{array}{r}
A_{i j}=\sum_{m=1}^{\infty} Y_{m}^{I} \frac{\tilde{Q}_{i}^{I}(m) \tilde{Q}_{j}^{I}(m)}{\sin \left(k_{z m}^{I} t\right)}, \\
C_{i j}=-\sum_{m=1}^{\infty} Y_{m}^{I} \frac{\tilde{Q}_{i}^{I}(m) \tilde{Q}_{j}^{I}(m)}{\tan \left(k_{z m}^{I} t\right)} \\
-\sum_{m=1}^{\infty} Y_{m}^{I I} \frac{\tilde{Q}_{i}^{I I}(m) \tilde{Q}_{j}^{I I}(m)}{\tan \left(k_{z m}^{I I} s\right)}
\end{array}
$$


and

$$
D_{i j}=\sum_{m=1}^{\infty} Y_{m}^{I I} \frac{\tilde{Q}_{i}^{I I}(m) \tilde{Q}_{j}^{I I}(m)}{\sin \left(k_{z m}^{I I} s\right)} .
$$

Equation (16) is not in a convenient form. We first eliminate the vector $[d]$ to get a reduced equation in terms of $[c]$ only, or

$$
[R][c]+[U][c] e^{\theta p}+[U]^{t}[c] e^{-\theta p}=0
$$

The matrices in this equation are given by

$$
[R]=A C^{-1} A+D C^{-1} D-C
$$

and

$$
[U]=A C^{-1} D, \quad[U]^{t}=D C^{-1} A .
$$

Let $\lambda=e^{\theta p}$ and note that $e^{-\theta p}=\frac{1}{\lambda}$, then we have the following eigenvalue problem

$$
[U]^{-1}[R] \lambda[c]+\lambda^{2}[c]+[U]^{-1}[U]^{t}[c]=0
$$

Let us introduce a vector $[v]$ of the same dimension as $[c]$ such that

$$
[v]=\lambda[c]
$$

The eigenvalue equation can finally be rewritten in the more convenient form

$$
\left[\begin{array}{cc}
U^{-1} R & U^{-1} U^{t} \\
-I & 0
\end{array}\right]\left[\begin{array}{l}
v \\
c
\end{array}\right]+\lambda\left[\begin{array}{l}
v \\
c
\end{array}\right]=0
$$

Here, $I$ is the identity matrix of order $M \times M$.

In the numerical solution the modes of the dielectrically loaded section are used as basis functions for simplicity. The last equation shows that the propagation constants of the Floquet modes are indeed determined from the classical eigenvalues of a matrix eigenvalue problem; standard software packages can be used to straightforwardly determine the dispersion of a large number of modes instead of iterative process which would result from a non-linear determinantal equation.

\section{Results}

To establish the validity of the approach, we determine the dispersion diagram of a structure where the dielectric constant of the disks approaches unity and compare with the analytical results of an empty circular waveguide. Figure 2 shows both numerical and analytical results with $\epsilon_{r}=1$. The agreement between the two is excellent. These results were obtained from 3 basis functions and 30 terms where used in computing the sums. Note that both diagrams are plotted using the reduced Brillouin zones [4].

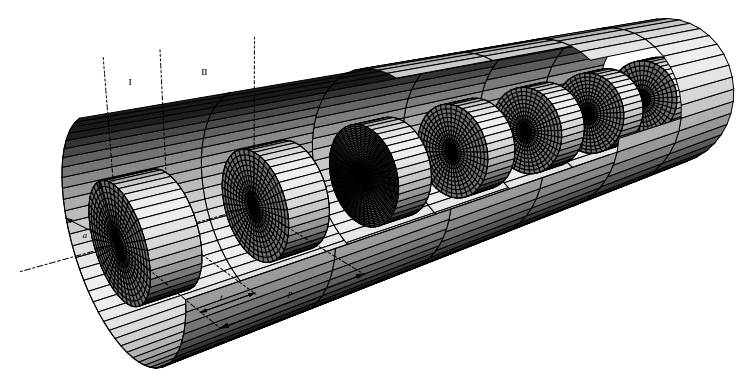

Fig. 1. Circular waveguide of radius a periodically loaded with dielectric disks (permittivity $\epsilon$, radius $b$ ) of thickness $t$. The period is $p$

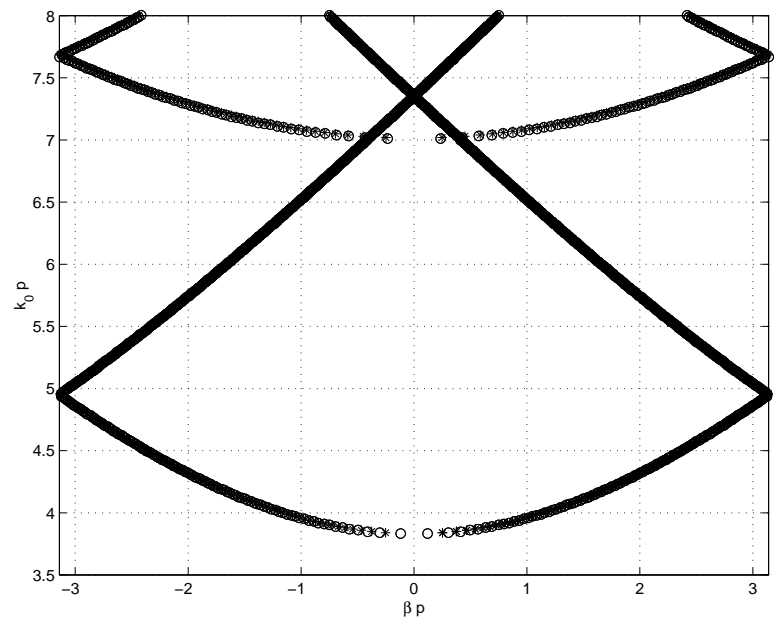

Fig. 2. $k_{0}-\beta$ diagram (circles) of the structure in Figure 1 when $\epsilon=1.01, b=0.5 \mathrm{a}, p=0.5$ and $t=0.5 p$. Also shown is the analytical $k_{0}-\beta$ diagram (*) of an empty circular waveguide of the same dimensions.

In the limit $t \rightarrow p$, the dispersion diagram of the periodic structure should approach that of a circular waveguide loaded with a dielectric rod of dielectric constant $\epsilon_{r}$. Figure 3 shows that the numerical results obtained when $t=0.99 p$ indeed satisfy this observation. 


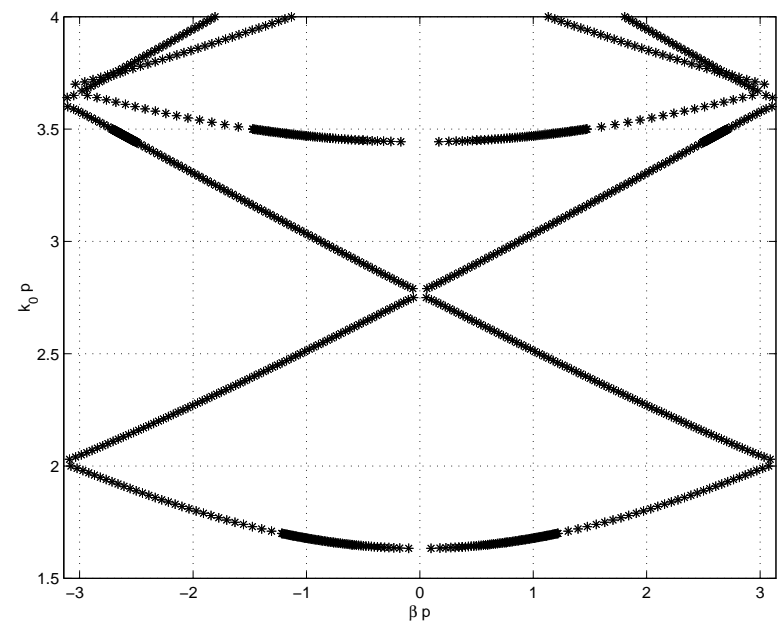

Fig. 3. $k_{0}-\beta$ diagram when $\epsilon=10, b=0.5 a, p=0.5$ and $t=0.99 p$. The diagram agrees very well with that of a circular waveguide loaded with a dielectric rod of the same characteristics.

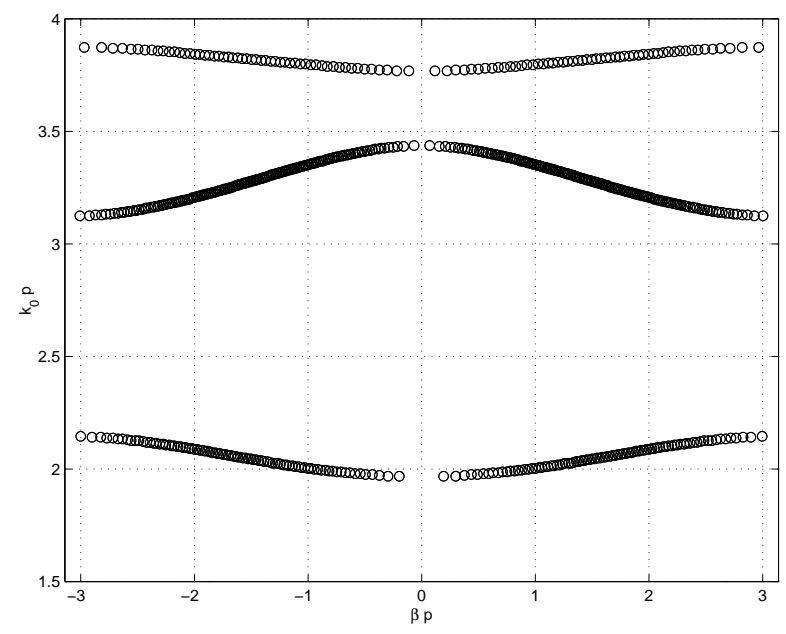

Fig. 4. $k_{0}-\beta$ diagram when $\epsilon=10, b=0.5 a, p=0.5$ a and $t=0.5 p$. The diagram agrees very well with that of a circular waveguide loaded with a dielectric rod of the same characteristics.

A more practical situation corresponding to a disk with dielectric constant $\epsilon_{r}=10$ was also analyzed; its $k_{0}-\beta$ diagram is shown in Figure 4. The presence of stop-bands separated by passbands is clearly visible. There is no propagation in the range of frequencies $2.144 \leq k_{0} p \leq 3.124$ and $3.436 \leq k_{0} p \leq 3.767$ although other modes with different angular dependence might be propagating in this frequency range. The effect of the dielectric loading on the dispersion diagram is also visible when compared with the empty waveguide of Figure 2. The addition of the dielectric disk lowers the cutoff frequency and flattens the branches of the dispersion diagram resulting in slowly changing group velocities. Although the first branch exhibits a positive group velocity, other higher branches support waves with negative group velocities which are essential to devices such as backward wave oscillators.

\section{Conclusions}

The propagation properties of angular independent modes in a circular waveguide periodically loaded with thick dielectric disks were accurately determined using the Coupled-Integral-Equation Technique (CIET). The propagation constants of the Floquet modes are determined from the classical eigenvalues of square matrix thereby considerably reducing CPU times. The approach allows efficient determination of propagating, evanescent as well as complex modes in this type of structures.

\section{References}

[1] R. E. Collin Field Theory of Guided Waves, IEEE Press, New York, 1991.

[2] P. J. B. Clarricoats and A. D. Olver Corrugated Horns for Microwave Antennas, Peregrinus, London, 1984.

[3] S. Amari, J. Bornemann and R. Vahldieck, Accurate analysis of scattering from multiple waveguide discontinuities using the coupled-integral-equation technique, Jou. Electromag. Waves Appl., vol. 10, pp.1623-1644, Dec. 1996.

[4] L. Brillouin, Wave Propagation in Periodic Structures, Dover, New York, 1953. 